Paper

\title{
On the covariance matrix of the stationary distribution of a noisy dynamical system
}

\author{
Makito Oku ${ }^{1,2 a)}$ and Kazuyuki Aihara ${ }^{1}$ \\ ${ }^{1}$ Institute of Industrial Science, The University of Tokyo \\ 4-6-1 Komaba, Meguro-ku, Tokyo 153-8505, Japan \\ ${ }^{2}$ Institute of Natural Medicine, University of Toyama \\ 2630 Sugitani, Toyama-shi, Toyama 930-0194, Japan \\ a) oku@inm.u-toyama.ac.jp
}

Received July 7, 2017; Revised December 10, 2017; Published April 1, 2018

\begin{abstract}
In this paper, we analyze the relation between the stability of a noisy dynamical system based on linear approximation and the covariance matrix of its stationary distribution. We reformulate the theory of dynamical network biomarkers in terms of the covariance matrix and clarify the limiting behavior of the covariance matrix when a dynamical system approaches a bifurcation point. We also discuss the relation between the Jacobian matrix and principal component analysis. An application to a simple nonlinear network model is also demonstrated.
\end{abstract}

Key Words: early warning signal, covariance matrix, Lyapunov equation, vector autoregressive model, multivariate Ornstein-Uhlenbeck process, principal component analysis

\section{Introduction}

Predicting state transitions is important in various fields such as ecology, economics, biology, medicine, and engineering $[1,2]$. Although the precise timing of the occurrence is usually difficult to predict, some early warning signals such as the increase in variance [3] or autocorrelation [4] of a state variable let us know the increase of possibility of the occurrence in near future.

From the viewpoint of dynamical systems theory, a stable fixed/equilibrium point with low stability has high risks of state transitions in two reasons. Here, we use the term stability in a broad sense regarding both the linear stability and basin sizes. One reason is that a small change of a parameter or parameters of the target system may cause a bifurcation, and thus the stable point loses its stability. Another reason is that external perturbations may push the system's state out of the basin boundary of the stable point. Therefore, evaluating the stability of dynamical systems is important to avoid undesirable system-wide events.

However, the precise mathematical expression of the dynamics is usually unknown for real-world complex systems, and thus we need to characterize the systems that have low stability from observations of the state variables. Many approaches have been investigated. The simplest way is based on the values of one or more state variables at a single time point. An anomaly of the target system is detected if a value is out of the predefined range. In addition, the sample mean values over multiple time points are also used to improve the reliability. However, this approach fails if the time series 
data of the observations has a slowly changing component and requires a detrending procedure [5]. In such a case, the mean values are shifted to be nearly constant. Moreover, the mean values does not change before certain types of bifurcations such as a Hopf bifurcation [6]. The second approach is based on model fitting $[7,8]$. The stability of the target system can be evaluated from the estimated parameters. However, this approach cannot be applied when the mathematical model to be fitted is unknown or the measurement intervals are too large. The third approach is based on the recovery rate [9-11]. This method requires to give a perturbation to the target system, and the system's stability is estimated from the relaxation process. Therefore, its usage is limited to the systems that can be easily perturbed.

Another approach which we focus on in this paper is based on the covariance matrix of the state variables and its related statistics including variance, standard deviation, and Pearson's correlation coefficient. The increase in the variance and the standard deviation of state variables has been observed in many real-world systems before imminent system-wide transitions $[1,3]$. The correlation coefficient between distinct state variables is also shown to serve as an indicator of the stability of multidimensional dynamical systems [12-16]. The increase in the largest eigenvalue of the covariance matrix has also been proposed as a possible early warning signal [17-19]. In addition, the eigenvector corresponding to the largest eigenvalue is also important to identify the state variables that contribute largely to abnormal fluctuations [15]. While the precise estimation of the covariance matrix from observations is difficult for high-dimensional systems [20], the estimation of only the largest eigenvalue and its corresponding eigenvector generally requires much less observations.

The relation between the covariance matrix and the stability of dynamical systems has long been investigated [21]. Especially, closed-form expressions of the covariance matrix based on the eigenvalues of the Jacobian matrix have been obtained both for discrete-time and continuous-time dynamical systems under general assumptions $[7,8]$. However, the limiting behavior of the variance, the standard deviation, and the correlation coefficient when a dynamical system approaches a bifurcation point has been only recently investigated in the context of early warning signals $[15,22]$. The original analysis in [15], which is called the dynamical network biomarker theory, was mainly for discretetime dynamical systems in which the dominant eigenvalue of the Jacobian matrix is real. Although the theory is also applicable to continuous-time systems by using time discretization, the converted Jacobian matrix explicitly depend on the discretized time step. In addition, the original analysis was based on correlation coefficient rather than covariance, and thus division into cases was needed because the denominator of the correlation coefficient can be zero. Another inconvenience of the correlation coefficient is that the analysis for the case of complex-valued dominant eigenvalues was complicated.

The contributions of this paper are as follows. First, we reformulate the theory in [15] in terms of the covariance matrix and reveal the limiting behavior of the covariance matrix when a dynamical system approaches a bifurcation point both for discrete-time and continuous-time systems. Our theory also fully covers the case where the dominant eigenvalues of the Jacobian matrix are a complex conjugate pair. Second, we derive simple expressions for the covariance matrix when the Jacobian matrix is normal or symmetric and noise is i.i.d. (independently and identically distributed). These simple expressions are useful for investigating the quantitative contributions of non-dominant eigenvalues to the covariance matrix. Although at least a part of those expressions have been obtained previously [19, 23], we would show that all of them can be derived from the discrete-time and continuous-time Lyapunov equations. Third, we discuss the relation between the eigenvalues and the eigenvectors of the Jacobian matrix and principal component analysis. This makes it much easier to identify largely contributing state variables, which are called dynamical network biomarkers [15] or dynamical network markers $[24,25]$.

The rest of the paper is organized as follows. In the subsequent four sections, we explain the findings of the theoretical analysis. Section 2 is for discrete-time linear dynamical systems, Section 3 is for continuous-time linear dynamical systems, Section 4 is for discrete-time nonlinear dynamical systems, and Section 5 is for continuous-time nonlinear dynamical systems. In Section 6, we apply the theory to an example model and show the results of numerical simulations. At last, we state our conclusions 
and discuss possible extensions in Section 7.

\section{Discrete-time linear dynamical systems}

\subsection{General case}

In this section, a discrete-time linear dynamical system of the following form is considered:

$$
x_{t+1}=A x_{t}+\xi_{t}, \quad \text { for } \quad t=0,1,2, \ldots,
$$

where $x_{t} \in \mathbb{R}^{n}$ is the state of the system at time $t, A \in \mathbb{R}^{n \times n}$ is a time-invariant matrix, and $\xi_{t}$ is noise at time $t$. In this paper, vectors are in column format by default. This system is also known as a vector autoregressive (VAR) model of order 1 [26]. The noise term is assumed to be zero-mean and independent of the system's state. Let $\lambda_{i} \in \mathbb{C}(i=1,2, \ldots, n)$ denote the eigenvalues of $A$. Then, we focus on the case where the spectral radius $\rho(A)=\max _{i}\left(\left|\lambda_{i}\right|\right)$ is less than 1 , that is, all the eigenvalues have magnitude less than 1 . In this case, the system's state fluctuates around the origin due to noise, and our goal is to clarify the relation between the stability of the system and the covariance matrix of the stationary distribution of $x$.

In the beginning, we explain the definition and some properties of covariance matrices. The covariance between real-valued random variables $X_{1}$ and $X_{2}$ is defined as $\operatorname{Cov}\left(X_{1}, X_{2}\right)=\mathrm{E}\left[\left(X_{1}-\right.\right.$ $\left.\left.\mathrm{E}\left(X_{1}\right)\right)\left(X_{2}-\mathrm{E}\left(X_{2}\right)\right)\right]$. Then, the covariance matrix of a $n$-dimensional real-valued random vector $X=\left(X_{1}, X_{2}, \ldots, X_{n}\right)^{T}$ is defined as the matrix whose $(i, j)$ element is $\operatorname{Cov}\left(X_{i}, X_{j}\right)$. A matrix $M \in \mathbb{C}^{n \times n}$ is said to be Hermitian if $M=M^{*}$, where $M^{*}=\bar{M}^{T}$ denote the conjugate transpose of $M$. A Hermitian matrix $M \in \mathbb{C}^{n \times n}$ is said to be positive-semidefinite and written as $M \succeq 0$ if, for any $v \in \mathbb{C}^{n}, v^{*} M v \geq 0$ holds. If strict inequality holds, $M$ is said to be positive-definite and written as $M \succ 0$. It is known that all covariance matrices are positivesemidefinite. When a covariance matrix is positive-definite, it can be converted to a correlation matrix whose $(i, j)$ element is the Pearson's correlation coefficient between $X_{i}$ and $X_{j}$ calculated as $\operatorname{Corr}\left(X_{i}, X_{j}\right)=\operatorname{Cov}\left(X_{i}, X_{j}\right) \operatorname{Cov}\left(X_{i}, X_{i}\right)^{-1 / 2} \operatorname{Cov}\left(X_{j}, X_{j}\right)^{-1 / 2}$. However, the opposite conversion from a correlation matrix to a covariance matrix is not always possible.

Let two real positive-definite matrices $C, D \in \mathbb{R}^{n \times n}$ denote the covariance matrices of the stationary distributions of $x$ and $\xi$, respectively. These matrices and $A$ are related as follows:

$$
\begin{aligned}
C & =\mathrm{E}\left(x_{t+1} x_{t+1}^{T}\right), \\
& =\mathrm{E}\left(\left(A x_{t}+\xi_{t}\right)\left(A x_{t}+\xi_{t}\right)^{T}\right), \\
& =\mathrm{E}\left(A x_{t} x_{t}^{T} A^{T}\right)+\mathrm{E}\left(A x_{t} \xi_{t}^{T}\right)+\mathrm{E}\left(\xi_{t} x_{t}^{T} A^{T}\right)+\mathrm{E}\left(\xi_{t} \xi_{t}^{T}\right), \\
& =A C A^{T}+D,
\end{aligned}
$$

where we use the fact that all of $\mathrm{E}\left(x_{t}\right), \mathrm{E}\left(\xi_{t}\right), \mathrm{E}\left(x_{t} \xi_{t}^{T}\right)$, and $\mathrm{E}\left(\xi_{t} x_{t}^{T}\right)$ are 0 . This equation is known as the discrete-time Lyapunov equation [27], and it is known to have a unique solution $C$ with $C \succ 0$ for any $D \succ 0$ if and only if $\rho(A)<1$. The same equation appears when $\xi_{t}$ is replaced with a control input $B u_{t}$ in which $B B^{T}=D$ and $u_{t} \in \mathbb{R}^{n}$. In that case, the solution $C$ is called the controllability Gramian.

Moreover, since $C$ is symmetric, Eq. (2) can be rewritten as a system of $n(n+1) / 2$ linear equations as follows:

$$
\begin{aligned}
C_{i j} & =\sum_{k, l} A_{i k} C_{k l} A_{l j}^{T}+D_{i j}, \\
& =\sum_{k} A_{i k} A_{j k} C_{k k}+\sum_{k<l}\left(A_{i k} A_{j l}+A_{i l} A_{j k}\right) C_{k l}+D_{i j}, \quad \text { for } \quad i \leq j .
\end{aligned}
$$

Therefore, if $A$ and $D$ are given, $C$ can be obtained by solving Eq. (3) numerically or symbolically, even if $A$ is non-diagonalizable. If we forget that $C$ is symmetric, Eq. (3) can also be rewritten by using the Kronecker product. A few alternative expressions of $C$ for general cases are also known [27] and presented in Appendix A. They are useful for some theoretical analyses such as investigating the 
limiting behavior explained in the next subsection. However, simpler expressions can be obtained for particular cases, which gives us deeper insight into the behavior of the system.

It should be noted that once $C$ is obtained, covariances of $x$ between different time steps can be calculated as follows:

$$
C(m)=\mathrm{E}\left(x_{t+m} x_{t}^{T}\right)=A^{m} C, \quad \text { for } \quad m=0,1,2, \ldots .
$$

\subsection{Case of diagonalizable matrix}

One useful case is where $A$ is diagonalizable, that is, $A$ can be diagonalized by an invertible matrix $P \in \mathbb{C}^{n \times n}$ as $A=P \Lambda P^{-1}$, where $\Lambda \in \mathbb{C}^{n \times n}$ is a diagonal matrix whose diagonal elements are the eigenvalues of $A$. The $k$ th column vector of $P$, which is denoted as $p_{k} \in \mathbb{C}^{n}$, is an eigenvector corresponding to the $k$ th eigenvalue. We assume that each column vector is normalized as $\left\|p_{k}\right\|=$ $\sqrt{p^{*} p}=1$. Thus, any pair of column vectors of $P$ corresponding to a pair of complex conjugate eigenvalues of $A$ is complex conjugate to each other.

Let us rewrite Eq. (2) as follows:

$$
C=A C A^{*}+D
$$

Then, by left-multiplying $P^{-1}$ and right-multiplying $\left(P^{*}\right)^{-1}=\left(P^{-1}\right)^{*}$ to the both side, we get

$$
\begin{aligned}
P^{-1} C\left(P^{*}\right)^{-1} & =\Lambda P^{-1} C\left(P^{-1}\right)^{*} \Lambda^{*}+P^{-1} D\left(P^{*}\right)^{-1}, \\
\tilde{C} & =\Lambda \tilde{C} \Lambda^{*}+\tilde{D},
\end{aligned}
$$

where $\tilde{C}=P^{-1} C\left(P^{*}\right)^{-1}$ and $\tilde{D}=P^{-1} D\left(P^{*}\right)^{-1}[7,8]$. The two matrices $\tilde{C}, \tilde{D} \in \mathbb{C}^{n \times n}$ are positivedefinite because for any invertible matrix $U \in \mathbb{C}^{n \times n}$ and any positive-definite matrix $V \in \mathbb{C}^{n \times n}$, $U^{*} V U \succ 0$ holds.

Since $\Lambda$ is diagonal, Eq. (6) can be solved in an element-wise manner as follows [7, 8]:

$$
\begin{aligned}
\tilde{C}_{i j} & =\lambda_{i} \bar{\lambda}_{j} \tilde{C}_{i j}+\tilde{D}_{i j}, \\
\tilde{C}_{i j} & =\frac{\tilde{D}_{i j}}{1-\lambda_{i} \bar{\lambda}_{j}} .
\end{aligned}
$$

This expression can be rewritten by using the Hadamard product (element-wise multiplication) as follows:

$$
\begin{aligned}
\tilde{C} & =L \circ \tilde{D}, \\
L_{i j} & =\frac{1}{1-\lambda_{i} \bar{\lambda}_{j}} .
\end{aligned}
$$

Thus, we obtain

$$
\begin{aligned}
C & =P \tilde{C} P^{*}, \\
& =P(L \circ \tilde{D}) P^{*}, \\
& =P\left(L \circ\left(P^{-1} D\left(P^{*}\right)^{-1}\right)\right) P^{*} .
\end{aligned}
$$

From this expression, we can see how the system behaves when $\rho(A)$ is close to 1 . If the dominant eigenvalue $\lambda_{d}$ that has the largest magnitude is real and its algebraic multiplicity is 1 (that is, $A$ has exactly one eigenvalue equals to $\lambda_{d}$ ), we get

$$
\lim _{\lambda_{d} \rightarrow \pm 1}\left(1-\lambda_{d}^{2}\right) C=\tilde{D}_{d d} p_{d} p_{d}^{T},
$$

where $p_{d} \in \mathbb{R}^{n}$ is the dominant eigenvector corresponding to $\lambda_{d}$. Here, $\tilde{D}_{d d}$ is real and positive because it is a diagonal element of a positive-definite matrix. We can see from Eq. (11) that the covariance matrix $C$ becomes proportional to the outer product of the dominant eigenvector with itself when $\lambda_{d}$ 
is close to 1 or -1 . In addition, all the off-diagonal elements of the correlation matrix approach either 1 or -1 unless $p_{d}$ contains any element that is strictly 0 . The case where $p_{d}$ contains 0 -elements has been investigated in [15].

Similarly, if the dominant eigenvalues are a complex conjugate pair $\lambda_{d}, \lambda_{e}=\bar{\lambda}_{d}$ and both have algebraic multiplicity 1 , we get

$$
\begin{aligned}
\lim _{\left|\lambda_{d}\right| \rightarrow 1}\left(1-\left|\lambda_{d}\right|^{2}\right) C & =\tilde{D}_{d d} p_{d} p_{d}^{*}+\tilde{D}_{e e} p_{e} p_{e}^{*} \\
& =2 \tilde{D}_{d d} \operatorname{Re}\left(p_{d} p_{d}^{*}\right) \\
& =2 \tilde{D}_{d d}\left(\operatorname{Re}\left(p_{d}\right) \operatorname{Re}\left(p_{d}\right)^{T}+\operatorname{Im}\left(p_{d}\right) \operatorname{Im}\left(p_{d}\right)^{T}\right)
\end{aligned}
$$

where $p_{d}, p_{e}=\bar{p}_{d} \in \mathbb{C}^{n}$ are the dominant eigenvectors corresponding to $\lambda_{d}$ and $\lambda_{e}$, respectively. Here, a relation $\tilde{D}_{d d}=\tilde{D}_{e e}$ is used. A proof of this is presented in Appendix B, in which the linear independence of $\operatorname{Re}\left(p_{d}\right)$ and $\operatorname{Im}\left(p_{d}\right)$ is also shown. We can see from Eq. (12) that $C$ becomes proportional to a rank-2 matrix determined only by the dominant eigenvectors when $\left|\lambda_{d}\right|=\left|\lambda_{e}\right| \approx 1$.

In fact, Eqs. (11) and (12) hold even if $A$ is nondiagonalizable. This can be seen from the general form of $C$ based on the Jordan normal form of $A$ shown in Appendix A.

\subsection{Case of normal matrix and i.i.d. noise}

The expression for $C$ shown in Eq. (10) can be further simplified when $A$ is normal (that is, $A A^{*}=$ $\left.A^{*} A\right)$ and the noise is i.i.d. In this case, $A$ can be diagonalized by a unitary matrix $P \in \mathbb{C}^{n \times n}$. Therefore, $P^{-1}=P^{*}$ holds. In addition, the covariance matrix of the noise term becomes $D=\sigma^{2} I$, where $\sigma>0$ is the standard deviation of noise and $I$ is an identity matrix of size $n$.

Under these assumptions, the covariance matrix of $x$ becomes as follows:

$$
\begin{aligned}
C & =P\left(L \circ\left(P^{*}\left(\sigma^{2} I\right) P\right)\right) P^{*} \\
& =\sigma^{2} P(L \circ I) P^{*} \\
& =\sigma^{2} P(I-\Lambda \bar{\Lambda})^{-1} P^{*} \\
& =\sigma^{2}\left(I-A A^{*}\right)^{-1} \\
& =\sigma^{2}\left(I-A A^{T}\right)^{-1}
\end{aligned}
$$

Especially, Eq. (13) is useful to investigate the contribution of each eigenvalue and eigenvector. Precisely, it can be rewritten as follows:

$$
C=\sigma^{2} \sum_{k=1}^{n} \frac{p_{k} p_{k}^{*}}{1-\left|\lambda_{k}\right|^{2}}
$$

It should be noted that complex eigenvectors always appear in complex conjugate pairs because $A$ is real. In addition, we have assumed that $\left\|p_{k}\right\|=1$ for all $k$. Thus, if $p_{k}$ is not a real vector, there exists $p_{l}$ such that $p_{l}=\bar{p}_{k}$ and $p_{k} p_{k}^{*}+p_{l} p_{l}^{*}=2 \operatorname{Re}\left(p_{k} p_{k}^{*}\right)$.

\subsection{Case of symmetric matrix and i.i.d. noise}

A symmetric matrix is a special case of a normal matrix. If $A$ is symmetric (that is, $A^{T}=A$ ), all of the vectors and the matrices given so far become real-valued, and thus the situation is much simplified. For i.i.d. noise, we can use the following three equivalent expressions:

$$
\begin{aligned}
C & =\sigma^{2} P\left(I-\Lambda^{2}\right)^{-1} P^{T}, \\
& =\sigma^{2} \sum_{k=1}^{n} \frac{p_{k} p_{k}^{T}}{1-\lambda_{k}^{2}}, \\
& =\sigma^{2}\left(I-A^{2}\right)^{-1} .
\end{aligned}
$$

A similar expression to the right hand side of Eq. (17) has been obtained in [23]. 
Among the three expressions, the first one is particularly important because it is of the form of the eigendecomposition of the covariance matrix $C$ in the real space. Therefore, it is related to principal component analysis (PCA) and singular value decomposition (SVD) [28], which are associated with the eigendecomposition of sample covariance matrices. Let $x_{1}, x_{2}, \ldots, x_{N} \in \mathbb{R}^{n}$ denote $N$ observations of a $n$-dimensional vector. For the case of a dynamical system, they are observations made at different time steps. Let $m \in \mathbb{R}^{n}$ denote the sample mean vector defined as $m=(1 / N) \sum_{k=1}^{N} x_{k}$. Then, the data matrix $X \in \mathbb{R}^{N \times n}$ is calculated as follows:

$$
X=\left[x_{1}-m, x_{2}-m, \ldots, x_{N}-m\right]^{T} .
$$

The sample covariance matrix $\hat{C} \in \mathbb{R}^{n \times n}$ corresponding to $X$ is defined as follows:

$$
\begin{aligned}
\hat{C} & =\frac{1}{N-1} X^{T} X \\
\hat{C}_{i j} & =\frac{1}{N-1} \sum_{k=1}^{N}\left(\left(x_{k}\right)_{i}-m_{i}\right)\left(\left(x_{k}\right)_{j}-m_{j}\right) .
\end{aligned}
$$

On the other hand, PCA is related to the eigendecomposition of $X^{T} X$ written as follows:

$$
X^{T} X=W R W^{T},
$$

where $W \in \mathbb{R}^{n \times n}$ is an orthogonal matrix whose columns are called weight vectors or loading vectors, and $R \in \mathbb{R}^{n \times n}$ is a diagonal matrix whose diagonal elements are nonnegative and are arranged in descending order. By using $W$, the data matrix $X$ is transformed as follows:

$$
T=X W,
$$

where $T \in \mathbb{R}^{N \times n}$ is called a score matrix in which the $k$ th column vector corresponds to the $k$ th principal component.

Usually, the eigendecomposition of $X^{T} X$ is not explicitly calculated, but SVD is performed instead as follows:

$$
X=U S W^{T},
$$

where $U \in \mathbb{R}^{N \times N}$ is another orthogonal matrix, and $S \in \mathbb{R}^{N \times n}$ is a rectangular diagonal matrix whose diagonal elements $s_{1} \geq s_{2} \geq \ldots \geq 0$ are called singular values. Since $R=S^{T} S$ holds, the eigenvalues of $X^{T} X$ are equal to the squared singular values.

When $N$ is sufficiently large and all the eigenvalues of $C$ are distinct, we can relate the analytical eigendecompositions of $C$ shown in Eq. (16) and the empirical eigendecomposition of $X^{T} X$ shown in Eq. (22). By comparing the two equations, we see that the $k$ th eigenvector of $A$ corresponds to the $k$ th loading vector, and the eigenvalues are related as follows:

$$
\frac{\sigma^{2}}{1-\lambda_{i}^{2}}=\frac{s_{i}^{2}}{N-1}, \quad \text { for } \quad i=1,2, \ldots, n
$$

Since $s_{i}^{2}$ increases in proportion to $N$ for large $N$, the right hand side does not go to 0 as $N \rightarrow \infty$. These relations indicate that we can estimate both the eigenvalues and the eigenvectors of $A$ from observations if we know that $A$ is symmetric and $D=\sigma^{2} I$.

In addition, the dominant eigenvectors can be estimated by using PCA and SVD for general $A$ with $\rho(A) \approx 1$ and $D \succ 0$. In the case of Eq. (11), most variance in data is explained by the first principal component, and the first loading vector approaches the dominant eigenvector $p_{d}$. This does not mean that the stationary distribution of $x$ shrinks in the other directions. Rather, it expands in the direction of $p_{d}$ so that its relative contribution to $C$ becomes dominant. In the case of Eq. (12), the first and the second principal components explain most variance, and the 2-dimensional linear subspace spanned by the first and the second loading vectors approaches the subspace spanned by $\operatorname{Re}\left(p_{d}\right)$ and $\operatorname{Im}\left(p_{d}\right)$. 


\section{Continuous-time linear dynamical systems}

\subsection{General case}

In this section, a continuous-time linear dynamical system of the following form is considered:

$$
\mathrm{d} x_{t}=A x_{t} \mathrm{~d} t+B \mathrm{~d} w_{t}, \quad \text { for } \quad t \geq 0,
$$

where $x_{t} \in \mathbb{R}^{n}$ is the state of the system at time $t, A, B \in \mathbb{R}^{n \times n}$ are time-invariant matrices, and $w_{t} \in \mathbb{R}^{n}$ is a vector of independent Wiener processes. This system is also known as a multivariate Ornstein-Uhlenbeck (OU) process [29]. Let $\lambda_{i} \in \mathbb{C}(i=1,2, \ldots, n)$ denote again the eigenvalues of $A$. Then, we assume that $\operatorname{Re}\left(\lambda_{i}\right)<0$ for all $\lambda_{i}$. In this case, $A$ is called an asymptotically stable matrix or a Hurwitz matrix.

This continuous-time dynamics can be converted to a discrete-time dynamics by using EulerMaruyama method [30] as follows:

$$
x_{t+\Delta t}=x_{t}+\Delta t A x_{t}+\sqrt{\Delta t} \xi_{t}, \quad \text { for } \quad t=0, \Delta t, 2 \Delta t, \ldots,
$$

where $\Delta t>0$ is a discretized time step, and $\xi_{t} \in \mathbb{R}^{n}$ is a random vector following $\mathcal{N}(0, D)$. Here, we set $D=B B^{T}$.

Let $C \in \mathbb{R}^{n \times n}$ denote again the covariance matrix of $x$. Then, we obtain the following relation:

$$
\begin{aligned}
\frac{\partial C}{\partial t} & =\lim _{\Delta t \rightarrow 0} \frac{\mathrm{E}\left(x_{t+\Delta t} x_{t+\Delta t}^{T}\right)-\mathrm{E}\left(x_{t} x_{t}^{T}\right)}{\Delta t}, \\
& =\lim _{\Delta t \rightarrow 0} \frac{\mathrm{E}\left(\left(x_{t}+\Delta t A x_{t}+\sqrt{\Delta t} \xi_{t}\right)\left(x_{t}+\Delta t A x_{t}+\sqrt{\Delta t} \xi_{t}\right)^{T}\right)-\mathrm{E}\left(x_{t} x_{t}^{T}\right)}{\Delta t}, \\
& =\lim _{\Delta t \rightarrow 0} \frac{\Delta t \mathrm{E}\left(A x_{t} x_{t}^{T}\right)+\Delta t \mathrm{E}\left(x_{t} x_{t}^{T} A^{T}\right)+(\Delta t)^{2} \mathrm{E}\left(A x_{t} x_{t}^{T} A^{T}\right)+\Delta t \mathrm{E}\left(\xi_{t} \xi_{t}^{T}\right)}{\Delta t}, \\
& =A C+C A^{T}+D=0,
\end{aligned}
$$

where we use the fact that all of $\mathrm{E}\left(x_{t}\right), \mathrm{E}\left(\xi_{t}\right), \mathrm{E}\left(x_{t} \xi_{t}^{T}\right)$, and $\mathrm{E}\left(\xi_{t} x_{t}^{T}\right)$ are 0 . This equation is known as the continuous-time Lyapunov equation [27], and it is known that $C$ with $C \succ 0$ uniquely exists for any $D \succ 0$ if and only if $A$ is asymptotically stable. The solution $C$ again becomes the controllability Gramian when the noise term is replaced with a control input. Since $C$ is symmetric, Eq. (28) can be rewritten as a system of $n(n+1) / 2$ linear equations as follows:

$$
\begin{aligned}
0 & =\sum_{k} A_{i k} C_{k j}+\sum_{k} C_{i k} A_{k j}^{T}+D_{i j}, \\
& =\sum_{k<j} A_{i k} C_{k j}+\sum_{k \geq j} A_{i k} C_{j k}+\sum_{k<i} C_{k i} A_{j k}+\sum_{k \geq i} C_{i k} A_{j k}+D_{i j}, \quad \text { for } \quad i \leq j .
\end{aligned}
$$

This system of linear equations can be rewritten by using the Kronecker product where all $n^{2}$ elements of $C$ are treated as unknown variables.

It should also be noted that covariances of $x$ at different time points can be written by using $C$ as follows [29]:

$$
C(s)=\mathrm{E}\left(x_{t+s} x_{t}^{T}\right)=\exp (A s) C, \quad \text { for } \quad s \geq 0,
$$

where $\exp (A s)$ is the matrix exponential of $A s$, that is, $\exp (A s)=\sum_{k=0}^{\infty}(A s)^{k} / k$ !. The equation above can be derived from $\partial C(s) / \partial s=A C(s)$.

\subsection{Case of diagonalizable matrix}

If matrix $A$ is diagonalizable, there exists an invertible matrix $P \in \mathbb{C}^{n \times n}$ satisfying $A=P \Lambda P^{-1}$, where $\Lambda \in \mathbb{C}^{n \times n}$ is diagonal. The column vectors of $P$ are the eigenvectors of $A$, and the diagonal elements of $\Lambda$ are the eigenvalues of $A$. The norm of each column vector of $P$ is normalized to 1 .

Let us rewrite Eq. (28) as follows: 


$$
A C+C A^{*}+D=0
$$

Then, by left-multiplying $P^{-1}$ and right-multiplying $\left(P^{*}\right)^{-1}=\left(P^{-1}\right)^{*}$ to the both side [7], we obtain

$$
\begin{aligned}
& \Lambda P^{-1} C\left(P^{*}\right)^{-1}+P^{-1} C\left(P^{-1}\right)^{*} \Lambda^{*}+P^{-1} D\left(P^{*}\right)^{-1}=0, \\
& \Lambda \tilde{C}+\tilde{C} \Lambda^{*}+\tilde{D}=0,
\end{aligned}
$$

where $\tilde{C}=P^{-1} C\left(P^{*}\right)^{-1}$ and $\tilde{D}=P^{-1} D\left(P^{*}\right)^{-1}$. The two matrices $\tilde{C}, \tilde{D} \in \mathbb{C}^{n \times n}$ are positive-definite again.

Since $\Lambda$ is diagonal, Eq. (32) can be solved in an element-wise manner as follows [7]:

$$
\begin{aligned}
& \lambda_{i} \tilde{C}_{i j}+\bar{\lambda}_{j} \tilde{C}_{i j}+\tilde{D}_{i j}=0, \\
& \tilde{C}_{i j}=-\frac{\tilde{D}_{i j}}{\lambda_{i}+\bar{\lambda}_{j}} .
\end{aligned}
$$

This equation can be rewritten by using the Hadamard product as follows:

$$
\begin{aligned}
\tilde{C} & =L \circ \tilde{D}, \\
L_{i j} & =-\frac{1}{\lambda_{i}+\bar{\lambda}_{j}} .
\end{aligned}
$$

Thus, we obtain

$$
\begin{aligned}
C & =P \tilde{C} P^{*}, \\
& =P(L \circ \tilde{D}) P^{*}, \\
& =P\left(L \circ\left(P^{-1} D\left(P^{*}\right)^{-1}\right)\right) P^{*} .
\end{aligned}
$$

Although this equation seems to be the same as Eq. (10), the definition of $L$ is different.

From this expression, we can see how the system behaves when $\max _{i} \operatorname{Re}\left(\lambda_{i}\right)$ is close to 0 . If the dominant eigenvalue $\lambda_{d}$ that has the largest real part is real and its algebraic multiplicity is 1 , we get

$$
\lim _{\lambda_{d} \rightarrow 0}\left(-2 \lambda_{d}\right) C=\tilde{D}_{d d} p_{d} p_{d}^{T}
$$

where $p_{d} \in \mathbb{R}^{n}$ is the dominant eigenvector corresponding to $\lambda_{d}$. Here, $\tilde{D}_{d d}$ is real and positive again because it is a diagonal element of a positive-definite matrix. We can see from Eq. (37) that the covariance matrix $C$ becomes proportional to the outer product of the dominant eigenvector with itself when $\operatorname{Re}\left(\lambda_{d}\right)$ is close to 0 .

Similarly, if the dominant eigenvalues are a complex conjugate pair $\lambda_{d}, \lambda_{e}=\bar{\lambda}_{d}$ and both have algebraic multiplicity 1 , we get

$$
\begin{aligned}
\lim _{\operatorname{Re}\left(\lambda_{d}\right) \rightarrow 0}\left(-2 \operatorname{Re}\left(\lambda_{d}\right)\right) C & =\tilde{D}_{d d} p_{d} p_{d}^{*}+\tilde{D}_{e e} p_{e} p_{e}^{*} \\
& =2 \tilde{D}_{d d} \operatorname{Re}\left(p_{d} p_{d}^{*}\right) \\
& =2 \tilde{D}_{d d}\left(\operatorname{Re}\left(p_{d}\right) \operatorname{Re}\left(p_{d}\right)^{T}+\operatorname{Im}\left(p_{d}\right) \operatorname{Im}\left(p_{d}\right)^{T}\right),
\end{aligned}
$$

where $p_{d}, p_{e}=\bar{p}_{d} \in \mathbb{C}^{n}$ are the dominant eigenvectors corresponding to $\lambda_{d}$ and $\lambda_{e}$, respectively. Here, the relation $\tilde{D}_{d d}=\tilde{D}_{e e}$ is used again. We can see from Eq. (38) that $C$ becomes proportional to a rank-2 matrix determined only by the dominant eigenvectors when $\operatorname{Re}\left(\lambda_{d}\right)=\operatorname{Re}\left(\lambda_{e}\right) \approx 0$.

In addition, Eqs. (37) and (38) also hold even when $A$ is not diagonalizable. This can be shown in a similar manner to the discrete-time case.

\subsection{Case of normal matrix and i.i.d. noise}

The expression for $C$ shown in Eq. (36) can be further simplified when $A$ is normal and $D=\sigma^{2} I$. Here, $P$ becomes unitary so that $P^{-1}=P^{*}$ holds. In this case, the covariance matrix of $x$ becomes as follows: 


$$
\begin{aligned}
C & =P\left(L \circ\left(P^{*}\left(\sigma^{2} I\right) P\right)\right) P^{*}, \\
& =\sigma^{2} P(L \circ I) P^{*} \\
& =-\sigma^{2} P(\Lambda+\bar{\Lambda})^{-1} P^{*} \\
& =-\sigma^{2}\left(A+A^{*}\right)^{-1} \\
& =-\sigma^{2}\left(A+A^{T}\right)^{-1}
\end{aligned}
$$

The third line can be rewritten as

$$
C=-\sigma^{2} \sum_{k=1}^{n} \frac{p_{k} p_{k}^{*}}{\lambda_{k}+\bar{\lambda}_{k}}
$$

where $p_{k} \in \mathbb{C}^{n}$ is the $p$ th column vector of matrix $P$.

\subsection{Case of symmetric matrix and i.i.d. noise}

If $A$ is symmetric and $D=\sigma^{2} I, C$ becomes as follows:

$$
\begin{aligned}
C & =-\frac{\sigma^{2}}{2} P \Lambda^{-1} P^{T}, \\
& =-\frac{\sigma^{2}}{2} \sum_{k=1}^{n} \frac{p_{k} p_{k}^{T}}{\lambda_{k}}, \\
& =-\frac{\sigma^{2}}{2} A^{-1},
\end{aligned}
$$

where both $P$ and $\Lambda$ are real. The third equation (44) is a special case of the expression for the covariance matrix over a time window derived in [19].

Relations to PCA and SVD described in Eqs. (19)-(24) are as follows. When the number of observations $N$ is sufficiently large and the eigenvalues of $C$ are all distinct, the $k$ th eigenvector of $A$ corresponds to the $k$ th loading vector, and the eigenvalues are related as follows:

$$
-\frac{\sigma^{2}}{2 \lambda_{i}}=\frac{s_{i}^{2}}{N-1}, \quad \text { for } \quad i=1,2, \ldots, n,
$$

where $s_{i}$ is the $i$ th singular value.

\section{Discrete-time nonlinear dynamical systems}

In this section, a discrete-time autonomous nonlinear dynamical system with additive noise is considered. It is described as follows:

$$
x_{t+1}=f\left(x_{t}\right)+\xi_{t}, \quad \text { for } \quad t=0,1,2, \ldots,
$$

where $x_{t} \in \mathbb{R}^{n}$ represents a state of the system at time $t, f: \mathbb{R}^{n} \rightarrow \mathbb{R}^{n}$ is a nonlinear map, and $\xi_{t}$ is noise at time $t$. We assume that if the noise term is removed, the system has an asymptotically stable (that is, Lyapunov stable and attracting [31]) fixed point $a \in \mathbb{R}^{n}$ that satisfies $f(a)=a$, and that $f$ is continuously differentiable at $a$. This means that $f$ is differentiable at $a$ and the derivative is continuous at $a$. We also assume that the fixed point $a$ is hyperbolic, that is, the Jacobian matrix of $f$ at $a$ has no eigenvalue on the unit circle in the complex plane. It should be noted that if the asymptotically stable fixed point is non-hyperbolic (for example, the origin of a one-dimensional map $x_{t+1}=\tanh x_{t}$ ), our knowledge described in Section 2 cannot be directly applied because the discrete-time Lyapunov equation (2) does not have a solution. The noise term is again assumed to be zero-mean and independent of the system's state. An additional assumption is that the noise intensity is sufficiently small so that we can perform the linear approximation of $f$ around $a$ as follows:

$$
f(x) \approx f(a)+J(a)(x-a),
$$

where $J(a) \in \mathbb{R}^{n \times n}$ is the Jacobian matrix of $f$ at $a$ defined as follows: 


$$
(J(a))_{i j}=\left.\frac{\partial f_{i}}{\partial x_{j}}\right|_{x=a} .
$$

Since $a$ is asymptotically stable and hyperbolic, $\rho(J(a))<1$ holds. By using the approximation, Eq. (46) becomes as follows:

$$
\begin{aligned}
& x_{t+1}=a+J(a)\left(x_{t}-a\right)+\xi_{t}, \\
& \tilde{x}_{t+1}=J(a) \tilde{x}_{t}+\xi_{t},
\end{aligned}
$$

where a new variable $\tilde{x}_{t}=x_{t}-a$ is introduced. Thus, all the findings in Section 2 can be applied.

There are three representative cases related to codimension- 1 bifurcations. The first case is just before a saddle-node bifurcation (also known as a fold bifurcation or a tangential bifurcation), in which a real eigenvalue is close to 1 . The second case is just before a period-doubling bifurcation, in which a real eigenvalue is close to -1 . The last case is right before a Neimark-Sacker bifurcation, where a pair of eigenvalues that are complex conjugate of each other are near the unit circle. The first and the second cases correspond to Eq. (11), and the third case corresponds to Eq. (12). Based on these equations, we can characterize the systems that have high risks of system-wide changes and estimate the dominant eigenvectors from observations.

\section{Continuous-time nonlinear dynamical systems}

In this section, a continuous-time autonomous nonlinear dynamical system with additive noise is considered. It is represented as follows:

$$
\mathrm{d} x_{t}=f\left(x_{t}\right) \mathrm{d} t+B \mathrm{~d} w_{t},
$$

The definition of $x_{t}$ and $f$ is the same to that in Section 4. The definition of $w_{t}$ and $B$ is the same to that in Section 3. We assume that the noiseless system $\dot{x}_{t}=f\left(x_{t}\right)$ has an asymptotically stable equilibrium point $a \in \mathbb{R}^{n}$ that satisfies $f(a)=0$, and that $f$ is continuously differentiable at $a$. We also assume that $a$ is hyperbolic, that is, the Jacobian matrix of $f$ at $a$ has no eigenvalue with the zero real part, and $a$ has no center manifold. An example of non-hyperbolic equilibrium points is the origin of a one-dimensional differential equation $\dot{x}_{t}=-x_{t}^{3}$. The assumption of hyperbolicity is needed in order for the continuous-time Lyapunov equation (28) to have a solution. Then, the linear approximation of $f$ around $a$ as shown in Eq. (47) gives

$$
\begin{aligned}
& \mathrm{d} x_{t}=J(a)\left(x_{t}-a\right) \mathrm{d} t+B \mathrm{~d} w_{t}, \\
& \mathrm{~d} \tilde{x}_{t}=J(a) \tilde{x}_{t} \mathrm{~d} t+B \mathrm{~d} w_{t},
\end{aligned}
$$

where a variable transformation $\tilde{x}_{t}=x_{t}-a$ is used. Here, all the eigenvalues of $J(a)$ have negative real parts because $a$ is asymptotically stable and hyperbolic. Thus, all the findings in Section 3 can be applied.

The relation between the Jacobian matrix $J(a)$ and the covariance matrix $C$ gives us a way to characterize the situation where at least one eigenvalue of $J(a)$ has a real part close to 0 . There are two representative cases. The first case is just before either a saddle-node bifurcation, a transcritical bifurcation, or a pitchfork bifurcation. In this case, one real eigenvalue is located near 0 . This case corresponds to Eq. (37). The second case is right before a Hopf bifurcation, in which two eigenvalues that are complex conjugate of each other have real parts close to 0 . This case corresponds to Eq. (38).

\section{Numerical simulations}

\subsection{Example model}

In this section, we demonstrate how to apply the theory to nonlinear dynamical systems in practice by using an example model. Let us consider a network system of $n$ nodes in which each node has its intrinsic dynamics and also interacts with its neighbors. The intrinsic dynamics of node $i$ is based on the following function: 


$$
F_{i}\left(x_{i}\right)=x_{i}-x_{i}^{3}-\mu_{i}
$$

where $x_{i} \in \mathrm{R}$ is the state of node $i$, and $\mu_{i} \in \mathrm{R}$ is a parameter. This function is the negative derivative of a double-well potential $U_{i}\left(x_{i}\right)=-x_{i}^{2} / 2+x_{i}^{4} / 4+\mu_{i} x_{i}$. The isolated dynamical system $\dot{x}_{i}=F_{i}\left(x_{i}\right)$ exhibits saddle-node bifurcations at $\mu_{i}= \pm 2 \sqrt{3} / 9$. We choose this model for simplicity, but more realistic ecological models with a few parameters are often used in the studies of early warning signals $[10,12,25,32-34]$.

Let $G=(V, E)$ denote the network structure of a random geometric graph [35] in which $V$ is the vertex set and $E \subseteq V \times V$ is the edge set. The node positions are randomly located in $[0,1]^{2}$, and two nodes are connected by an edge if the Euclidean distance between them is less than or equal to the threshold $d$. Let $L_{G} \in \mathbb{Z}^{n \times n}$ denote the Laplacian matrix of $G$. $L_{G}$ is a symmetric matrix whose $i$ th diagonal element is the degree of node $i$ (that is, the number of edges incident to node $i$ ), and $(i, j)$ element for $i \neq j$ is -1 if node $i$ and node $j$ are adjacent and 0 otherwise.

Then, the dynamics of the network system is described by the following stochastic differential equation:

$$
\mathrm{d} x(t)=[F(x(t))+\alpha M x(t)] \mathrm{d} t+B \mathrm{~d} w(t),
$$

where $x=\left(x_{1}, \ldots, x_{n}\right)^{T}$ is the state of the system, $F(x)=\left(F_{1}\left(x_{1}\right), \ldots, F_{n}\left(x_{n}\right)\right)^{T}$ corresponds to the intrinsic dynamics, $\alpha \geq 0$ is the coupling strength, $M=-L_{G}$ is the sign-reversed Laplacian matrix of $G$, and $w \in \mathbb{R}^{n}$ is a vector of independent Wiener processes. It should be noted that the $i$ th element of $M x(t)$ can be rewritten as $\sum_{j} M_{i j} x_{j}(t)=\sum_{j,(i, j) \in E}\left(x_{j}(t)-x_{i}(t)\right)$, which is known as diffusive coupling. The covariance matrix of noise $D=B B^{T}$ is defined as $D_{i j}=\sigma^{2} \exp \left(-\gamma\left\|r_{i}-r_{j}\right\|^{2}\right)$, where $\sigma, \gamma>0$ are parameters, and $r_{i} \in[0,1]^{2}$ represents the position of node $i$. This means that close nodes receive much correlated noise than distant nodes. $B$ is calculated from $D$ by using the Cholesky decomposition. It should be noted that $D \succ 0$ holds because it is a Gram matrix of the Gaussian kernel [36].

We induce system-wide state transitions in this network model according to the following procedure. Let $x_{\mathrm{on}}$ and $x_{\mathrm{off}}$ denote the higher and the lower stable equilibrium points of the isolated system $\dot{x}_{i}=F_{i}\left(x_{i}\right)$, respectively. Initially, all nodes are set to $x_{\mathrm{on}}$. Then, in each iteration, we randomly select a node that has not been selected yet, deactivate it by setting its value to $x_{\text {off }}$, and update $a$ with the stable equilibrium point that the system without noise reaches eventually. Let $n_{\text {off }}$ denote the number of deactivated nodes. This does not include the nodes that are not selected but fall down due to interaction.

\subsection{Calculation of the covariance matrix}

The theoretical values of the covariance matrix $C$ is obtained in three steps. First, we need to numerically calculate the position of a stable equilibrium point $a$ that satisfies $F(a)+\alpha M a=0$. Next, the Jacobian matrix at $a$ is calculated as

$$
J(a)=\operatorname{diag}\left(\frac{\partial F_{1}\left(a_{1}\right)}{\partial x_{1}}, \ldots, \frac{\partial F_{n}\left(a_{n}\right)}{\partial x_{n}}\right)+\alpha M .
$$

Here, $\partial F_{i}\left(a_{i}\right) / \partial x_{i}=1-3 a_{i}^{2}$. Finally, $C$ is calculated according to Eq. (36).

On the other hand, the sample covariance matrix $\hat{C}$ is obtained as follows. First, we need to know again the position of a stable equilibrium point $a$. Next, a trajectory of the stochastic differential equation (53) is calculated numerically. In this example, we use the Euler-Maruyama method [30] to discretize time as follows:

$$
x(\tau+1)=x(\tau)+\Delta t[F(x(\tau))+\alpha M x(\tau)]+\sqrt{\Delta t} B \epsilon(\tau), \quad \text { for } \quad \tau=0,1,2, \ldots, \tau_{\max },
$$

where we set $\tau=t / \Delta t$, and $\epsilon(\tau)$ is a random vector following $\mathcal{N}(0, I)$. The initial state $x(0)$ is set to $a$. Since the multivariate OU process is correlated in the time domain, we only take $N$ points each of which is separated by $\tau_{\max } / N$ steps. Next, a data matrix $X$ is constructed according to Eq. (19). Then, the sample covariance matrix is calculated according to Eq. (20). 


\subsection{Simulation results}

In the following simulations, we set $\mu_{i}=0.25$ for all $i$. Other parameters are $n=100, d=0.25$, $\alpha=0.02, \sigma=0.001, \gamma=10, \Delta t=0.01, \tau_{\max }=10^{5}$, and $N=500$.

Figure 1 shows an example of system-wide state transitions in the network model. In this case, most nodes fall down at $n_{\mathrm{off}}=13$. Since the precise timing varies according to the node positions and the order of the node selection, we focus on this particular case henceforth. Notice that the mean value of $a$ clearly decreases until $n_{\text {off }}=12$, and thus the phenomenon is possibly used as an early warning signal, too.

Figure 2 shows the covariance matrix at the beginning $\left(n_{\text {off }}=0\right)$ and right before the system-wide state transition $\left(n_{\text {off }}=12\right)$. The theoretical predictions and the results of the numerical simulations

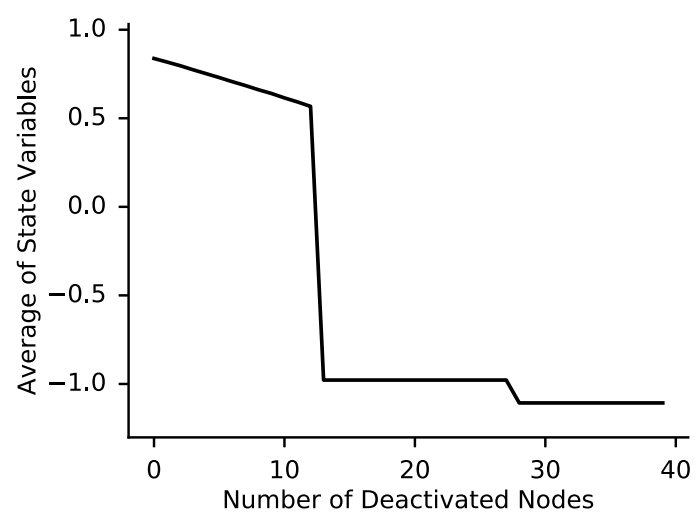

Fig. 1. Relation between the number of deactivated nodes $\left(n_{\text {off }}\right)$ and the average of the state variables evaluated at $a$.

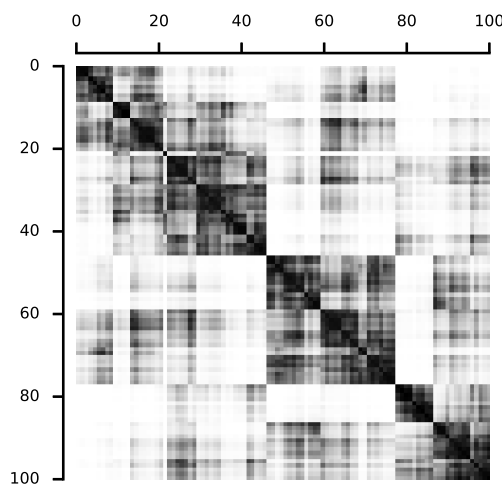

(A) Theory ( $n \_$off $=0$ )

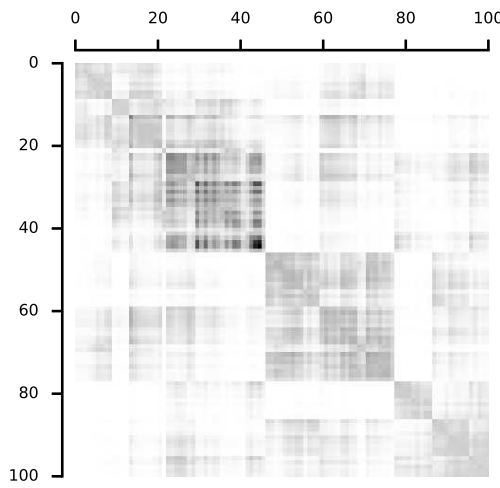

(C) Theory (n_off=12)

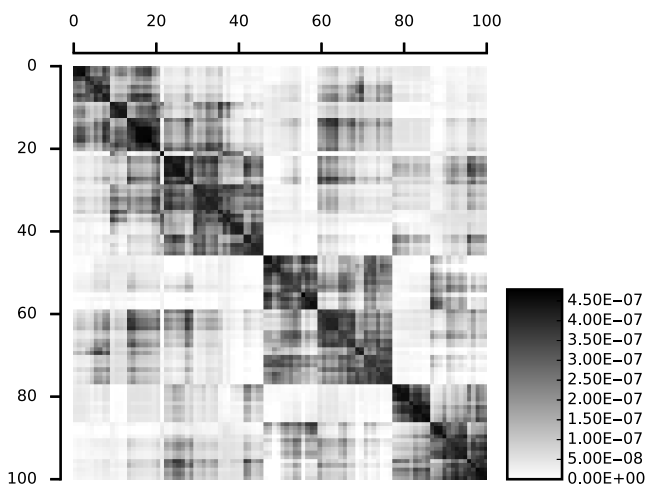

(B) Simulation (

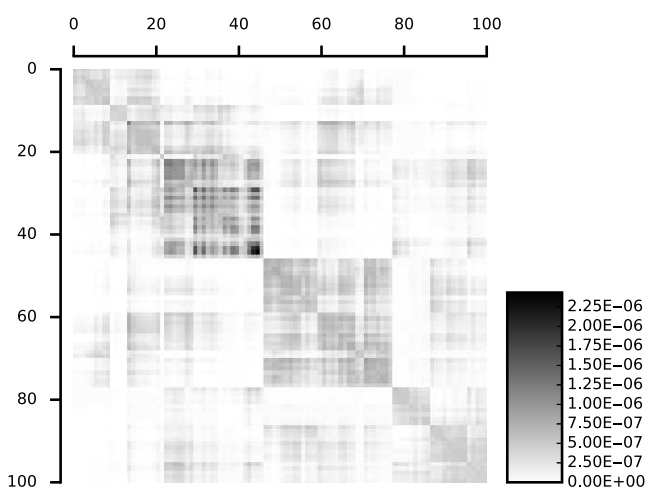

(D) Simulation (n_off $=12$ )

Fig. 2. Covariance matrices of the example model. (A) $C$ at $n_{\text {off }}=0$, (B) $\hat{C}$ at $n_{\text {off }}=0,(\mathrm{C}) C$ at $n_{\text {off }}=12$, and (D) $\hat{C}$ at $n_{\text {off }}=12$. The nodes are sorted based on the Euclidean distance between their positions by using hierarchical clustering with the average linkage method. 
match well in both cases. At $n_{\text {off }}=12$, a part of nodes show particularly high variances and also high covariances between them. Notice that the maximum values of the color scales in Figs. 2(C) and 2(D) are several times larger than those in Figs. 2(A) and 2(B).

On the other hand, it would be beneficial if a statistic can characterize the change of the covariance matrix. As an example, Fig. 3 shows the change in the largest eigenvalue of the covariance matrix. Its value tends to increase until $n_{\text {off }}=12$ both in theory and in the numerical simulations, although the simulation result is noisy. This supports the idea that the increase in the largest eigenvalue of the sample covariance matrix, which can be calculated only from measurements of the system, may serve as an early warning signal [17-19].

Another important aspect provided by the covariance matrix is which nodes contribute largely to the fluctuation just before the state transition. Figure 4 shows the state of the network at $n_{\text {off }}=12$. It can be seen that many active nodes (close to $x_{\text {on }}$ ) around the five inactive nodes (close to $x_{\text {off }}$ ) at the bottom right region show large contribution. In addition, high degree nodes tend to have large contribution in this model. This kind of information may help to take countermeasures for preventing system-wide changes.

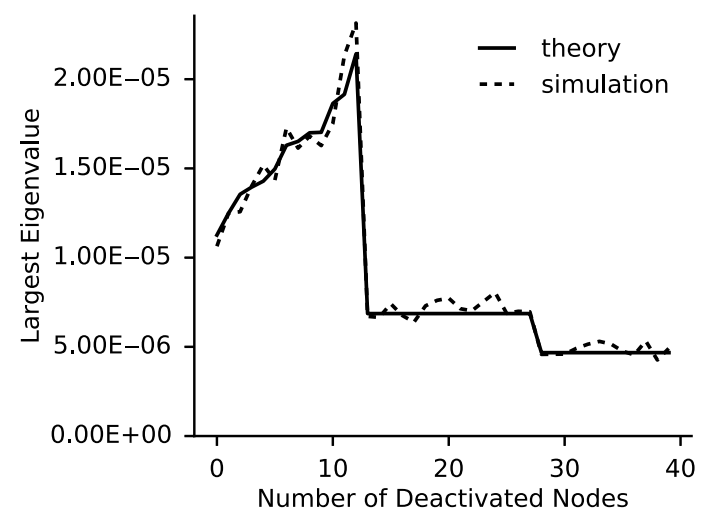

Fig. 3. Relation between the number of deactivated nodes $\left(n_{\text {off }}\right)$ and the largest eigenvalue of the covariance matrix.

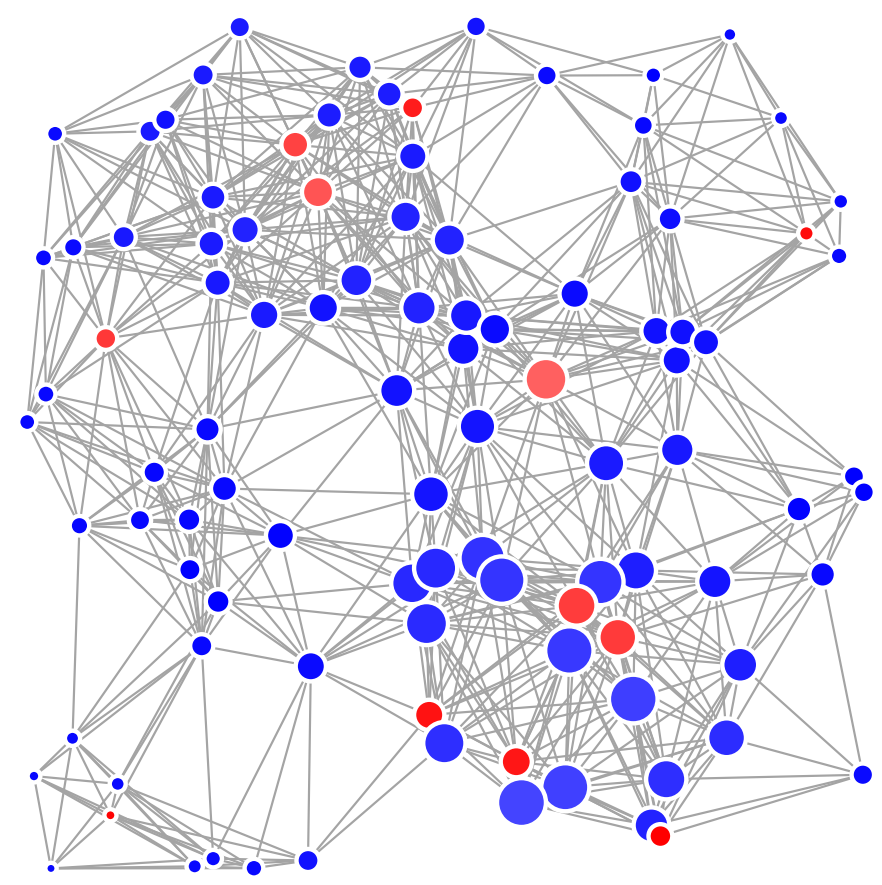

Fig. 4. Network view of the example model at $n_{\text {off }}=12$. Blue nodes are active (close to $x_{\mathrm{on}}$ ) and red nodes are inactive (close to $x_{\mathrm{off}}$ ). The diameter of each node is proportional to the absolute value of the corresponding element of the dominant eigenvector of $C$. 


\section{Discussion and Conclusions}

In this paper, we have investigated how the covariance matrix of the stationary distribution of the state of a dynamical system is associated with the eigenvalues and the eigenvectors of the Jacobian matrix of the system. Our major contributions are as follows. First, we have reformulated the theory in [15] in terms of the covariance matrix and revealed the asymptotic behavior of the covariance matrix when a dynamical system approaches a bifurcation point both for discrete-time and continuous-time cases, and both for real-valued and complex-valued dominant eigenvalues. Second, we have derived several simple expressions for the covariance matrix from the discrete-time and continuous-time Lyapunov equations when the Jacobian matrix is normal or symmetric and noise is i.i.d. Third, we have pointed out that the eigenvalues and the eigenvectors of the Jacobian matrix correspond to the singular values of SVD and the loading vectors of PCA, respectively, when the Jacobian matrix is symmetric and noise is i.i.d. In addition, we have also applied the theory to a nonlinear network model and demonstrated how the covariance matrix changes before a system-wide state transition.

We make some remarks regarding the extension of this work. First, we only considered dynamical noise that perturbs the true state of the system but did not consider observation noise that only distorts the measurement. However, the effect of observation noise in its simplest form is easy to incorporate into the theory. Let $y_{t}=x_{t}+\eta_{t}$ denote the observation at time $t$ where $\eta_{t}$ is the observation noise independent of $x_{t}$. Then, the covariance matrix of the stationary distribution of $y_{t}$ is simply the summation of the covariance matrices of $x_{t}$ and $\eta_{t}$.

Second, the current theory is based on linear approximation and cannot be used when noise is large or the asymptotically stable fixed/equilibrium point is non-hyperbolic. This is because the linear approximation as shown in Eq. (47) is based on a multivariate Taylor expansion of a nonlinear map $f$ at a stable fixed/equilibrium point $a$, and the higher order terms are neglected. Let us assume here that $a$ is hyperbolic for simplicity. Then, this approximation is accurate as long as the state variable $x$ stays close to $a$ so that the higher order terms are negligible. However, the accuracy becomes worse as $x$ moves away from $a$ because the influence of the higher order terms becomes large. Therefore, the theory based on the linear approximation cannot be applied to the cases where the position of $x$ is disturbed by large noise [37].

In that case, the stationary distribution is no longer a normal distribution, and we need to take into account the effect of nonlinearity. Analytic expressions of the stationary distribution have been obtained for particular cases by solving the Fokker-Planck equation $[38,39]$. In addition, the controllability Gramian which corresponds to the covariance matrix of an uncontrolled system has been extended to incorporate nonlinear dynamics [40]. The relative size of the basin of attraction [41] and its extension to finite retention time [42] are also important aspects to characterize the stability of dynamical systems. A phenomenon called flickering can also occur when noise is large $[34,43]$. Integrating these knowledge to construct a robust indicator for predicting system-wide changes in complex systems is a challenging future problem.

Other directions of future works regarding the theoretical underpinning include state-dependent noise, time delay, nonstationarity, transient dynamics, and other types of attractors such as periodic orbits. On the other hand, development of indicators both for general and specific purposes is also important.

Last but not least, practical applications of the theoretical framework refined in this study is a matter of high priority. Although the original theory in [15] has been already applied to several real data such as type 1 diabetes [44], type 2 diabetes [45], DNA methylation [46], foreign exchange market [24], and cell differentiation [47, 48], it is our important future work to utilize our new findings to broaden the applicability of the theory.

\section{Acknowledgments}

We would like to thank Dr. Koji Iwayama for pointing out the relation between dynamical network markers and principal component analysis. We would also like to thank Dr. Yuji Okamoto for suggesting counterparts in the field of control theory. This research is partially supported by JSPS 
KAKENHI Grant No. JP15H05707.

\section{Appendix}

\section{A. Alternative forms of $C$}

Here, we present a few alternative expressions of $C$ as the solution of the discrete-time Lyapunov equation (2) for $\rho(A)<1$ and $D \succ 0$. The first one is useful to see that Eqs. (11) and (12) hold in general. Let $\Lambda \in \mathbb{C}^{n \times n}$ be a Jordan normal form of $A$ that satisfies $A=P \Lambda P^{-1}$, where $P \in \mathbb{C}^{n \times n}$ is an invertible matrix. Each column vector of $P$ is a generalized eigenvector of $A$, and we assume that their norms are normalized to 1 . It should be noted that complex generalized eigenvectors always appear in complex conjugate pairs. $\Lambda$ is a block diagonal matrix written as follows:

$$
\begin{aligned}
\Lambda & =\left[\begin{array}{llll}
\Lambda_{1} & & & \\
& \Lambda_{2} & & \\
& & \ddots & \\
& & & \Lambda_{m}
\end{array}\right], \\
\Lambda_{k} & =\left[\begin{array}{cccc}
\lambda_{k} & 1 & & \\
& \lambda_{k} & \ddots & \\
& & \ddots & 1 \\
& & & \lambda_{k}
\end{array}\right], \text { for } k=1,2, \ldots, m,
\end{aligned}
$$

where $m$ is the number of the blocks and $\lambda_{k}$ is the eigenvalue related to the $k$ th block. Let $n(k)$ denote the size of $\Lambda_{k}$. From similar calculations to Eq. (6), we get

$$
\tilde{C}=\Lambda \tilde{C} \Lambda^{*}+\tilde{D},
$$

where $\tilde{C} \in \mathbb{C}^{n \times n}$ and $\tilde{D} \in \mathbb{C}^{n \times n}$ satisfy $C=P \tilde{C} P^{*}$ and $D=P \tilde{D} P^{*}$. Let us partition $\tilde{C}$ and $\tilde{D}$ into blocks as follows:

$$
\begin{aligned}
\tilde{C} & =\left[\begin{array}{cccc}
C_{11} & C_{12} & \cdots & C_{1 m} \\
C_{21} & C_{22} & \cdots & C_{2 m} \\
\vdots & \vdots & \ddots & \vdots \\
C_{m 1} & C_{m 2} & \cdots & C_{m m}
\end{array}\right], \\
\tilde{D} & =\left[\begin{array}{cccc}
D_{11} & D_{12} & \cdots & D_{1 m} \\
D_{21} & D_{22} & \cdots & D_{2 m} \\
\vdots & \vdots & \ddots & \vdots \\
D_{m 1} & D_{m 2} & \cdots & D_{m m}
\end{array}\right],
\end{aligned}
$$

where submatrices $C_{k l}$ and $D_{k l}$ have $n(k)$ rows and $n(l)$ columns. By using them, Eq. (A-3) can be rewritten in a blockwise manner as follows [27]:

$$
C_{k l}=\Lambda_{k} C_{k l} \Lambda_{l}^{*}+D_{k l}, \quad \text { for } \quad 1 \leq k, l \leq m .
$$

Thus, we can solve it for each block separately. Element-wise equations are given as follows [27]:

$$
\begin{array}{r}
\left(C_{k l}\right)_{i j}=\lambda_{k} \bar{\lambda}_{l}\left(C_{k l}\right)_{i j}+\lambda_{k}\left(C_{k l}\right)_{i, j+1}+\bar{\lambda}_{l}\left(C_{k l}\right)_{i+1, j}+\left(C_{k l}\right)_{i+1, j+1}+\left(D_{k l}\right)_{i j}, \\
\text { for } 1 \leq i \leq n(k), 1 \leq j \leq n(l),
\end{array}
$$

where we define $\left(C_{k l}\right)_{n(k)+1, j}=\left(C_{k l}\right)_{i, n(l)+1}=\left(C_{k l}\right)_{n(k)+1, n(l)+1}=0$. It can be seen that $\left(C_{k l}\right)_{i j}$ is determined if $\lambda_{k} \bar{\lambda}_{l} \neq 1$ and all of $\left(C_{k l}\right)_{i, j+1},\left(C_{k l}\right)_{i+1, j}$, and $\left(C_{k l}\right)_{i+1, j+1}$ have been calculated. Since $\left|\lambda_{k}\right|<1$ for all $k$ and $\left(C_{k l}\right)_{i j}$ values can be determined iteratively from the right and the bottom edges, all elements of $C_{k l}$ can be calculated. Moreover, all elements of $C_{k l}$ are rational functions of $\lambda_{k}$ and $\bar{\lambda}_{l}$, and their denominators are powers of $\left(1-\lambda_{k} \bar{\lambda}_{l}\right)$. Since every $C_{k l}$ is determined similarly, $\tilde{C}$ is constructed by combining them, and the solution of Eq. (2) is obtained as $C=P \tilde{C} P^{*}$. 
Next, we explain another expression for $C$, which is also valuable for some analysis. From Eq. (2), we get the following expression:

$$
\begin{aligned}
C & =A C A^{T}+D, \\
& =A\left(A C A^{T}+D\right) A^{T}+D, \\
& =A^{m} C\left(A^{T}\right)^{m}+D+A D A^{T}+\ldots+A^{m-1} D\left(A^{T}\right)^{m-1}, \quad \text { for } \quad m=1,2, \ldots
\end{aligned}
$$

By taking the limit of $m \rightarrow \infty$, the first term vanishes because all the eigenvalues of $A$ have magnitude less than 1 (for a rigorous proof of this, see [49]). Thus, $C$ becomes a series written as follows:

$$
C=\sum_{m=0}^{\infty} A^{m} D\left(A^{T}\right)^{m} .
$$

We already know that this converges because otherwise $C$ cannot be determined. From this expression, it can be seen that $C$ is real and positive-definite because (i) $D$ is real and positive-definite, (ii) $A$ is real, (iii) for any matrix $U \in \mathbb{C}^{n \times k}$ and any positive-definite matrix $V \in \mathbb{C}^{n \times n}, U^{*} V U \succeq 0$ holds, and (iv) a summation of a positive-definite matrix and a positive-semidefinite matrix is positive-definite.

Similarly, alternative expressions of $C$ as the solution of the continuous-time Lyapunov equation (28) are also known. A blockwise expression based on the Jordan normal form of $A$ can be similarly calculated. From it, we can see that Eqs. (37) and (38) hold in general. The expression shown in Eq. (A-11), which is based on a summation over infinite number of matrices, becomes an integral over an infinite range as follows [27]:

$$
C=\int_{0}^{\infty} \exp \left(A t^{\prime}\right) D \exp \left(A^{T} t^{\prime}\right) \mathrm{d} t^{\prime} .
$$

\section{B. Proof of $\tilde{D}_{d d}=\tilde{D}_{e e}$}

Here, we provide a proof for $\tilde{D}_{d d}=\tilde{D}_{e e}$.

Let $S_{1}=\left\{p_{1}, \ldots, p_{m}, \bar{p}_{1}, \ldots, \bar{p}_{m}, w_{1}, \ldots, w_{n-2 m}\right\}$ denote a set of $n$-dimensional vectors, and let $i$ denote the imaginary unit only in this section. The first $2 m$ vectors are complex-valued written as $p_{k}=u_{k}+i v_{k} \in \mathbb{C}^{n}\left(u_{k}, v_{k} \in \mathbb{R}^{n}\right)$. The remaining vectors are real-valued, that is, $w_{k} \in \mathbb{R}^{n}$. Let $S_{2}=\left\{u_{1}, \ldots, u_{m}, v_{1}, \ldots, v_{m}, w_{1}, \ldots, w_{n-2 m}\right\}$ denote another set of vectors, in which all element are real vectors.

Lemma. If the vectors in $S_{1}$ are linearly independent, then the vectors in $S_{2}$ are linearly independent.

Proof. Suppose that the vectors in $S_{2}$ are not linearly independent. Then, there exists a set of real-valued coefficients $a_{k}, b_{k}$, and $c_{k}$ that are not all zero, and

$$
\sum_{k=1}^{m}\left(a_{k} u_{k}+b_{k} v_{k}\right)+\sum_{k=1}^{n-2 m} c_{k} w_{k}=0
$$

holds. However, this leads to the following equality:

$$
\sum_{k=1}^{m}\left(\left(\frac{a_{k}-i b_{k}}{2}\right)\left(u_{k}+i v_{k}\right)+\left(\frac{a_{k}+i b_{k}}{2}\right)\left(u_{k}-i v_{k}\right)\right)+\sum_{k=1}^{n-2 m} c_{k} w_{k}=0 .
$$

This contradicts the supposition that the vectors in $S_{1}$ are linearly independent.

Next, let us consider a linear combination of the vectors in $S_{1}$ :

$$
q=\sum_{k=1}^{m}\left(\left(a_{k}+i b_{k}\right)\left(u_{k}+i v_{k}\right)+\left(c_{k}+i d_{k}\right)\left(u_{k}-i v_{k}\right)\right)+\sum_{k=1}^{n-2 m}\left(e_{k}+i f_{k}\right) w_{k},
$$

where $a_{k}, b_{k}, c_{k}, d_{k}, e_{k}$, and $f_{k}$ are real coefficients. Assume that the vectors in $S_{1}$ are linearly independent. When $q$ is real, we obtain 


$$
\operatorname{Im}(q)=\sum_{k=1}^{m}\left(\left(b_{k}+d_{k}\right) u_{k}+\left(a_{k}-c_{k}\right) v_{k}\right)+\sum_{k=1}^{n-2 m} f_{k} w_{k}=0 .
$$

Since $u_{k}, v_{k}$, and $w_{k}$ are linearly independent from the lemma, $b_{k}=-d_{k}, a_{k}=c_{k}$, and $f_{k}=0$ hold for all $k$. This means that, if a linear combination gives a real value, every pair of coefficients corresponding to a complex conjugate vector is complex conjugate to each other.

Let us introduce a new variable $\zeta \in \mathbb{C}^{n}$ that satisfies $P \zeta=\xi$ and $\mathrm{E}\left(\zeta \zeta^{*}\right)=P^{-1} D\left(P^{-1}\right)^{*}=\tilde{D}$. Each column vector of $P$ corresponds to an eigenvector of $A$, and we have assumed that each column vector's norm is normalized to 1 . Since $A$ is real, all complex eigenvectors appear in complex conjugate pairs. Moreover, since $P$ is invertible, its column vectors are linearly independent. Thus, the discussion above can be applied by regarding the column vectors of $P$ as $S_{1}$. Let $\zeta_{d}$ and $\zeta_{e}$ denote the two elements corresponding to the dominant complex eigenvalues of $A$. Since $\xi$ is real, they are complex conjugate to each other. Thus, we obtain

$$
\tilde{D}_{d d}=\mathrm{E}\left(\zeta_{d} \bar{\zeta}_{d}\right)=\mathrm{E}\left(\bar{\zeta}_{d} \zeta_{d}\right)=\mathrm{E}\left(\zeta_{e} \bar{\zeta}_{e}\right)=\tilde{D}_{e e}
$$

\section{References}

[1] M. Scheffer, J. Bascompte, W.A. Brock, V. Brovkin, S.R. Carpenter, V. Dakos, H. Held, E.H. van Nes, M. Rietkerk, and G. Sugihara, "Early-warning signals for critical transitions," Nature, vol. 461, pp. 53-59, September 2009. doi: 10.1038/nature08227.

[2] M. Scheffer, S.R. Carpenter, T.M. Lenton, J. Bascompte, W. Brock, V. Dakos, J. van de Koppel, I.A. van de Leemput, S.A. Levin, E.H. van Nes, M. Pascual, and J. Vandermeer, "Anticipating critical transitions," Science, vol. 338, pp. 344-348, October 2012. doi: 10.1126/science.1225244.

[3] S.R. Carpenter and W.A. Brock, "Rising variance: A leading indicator of ecological transition," Ecol. Lett., vol. 9, pp. 311-318, March 2006. doi: 10.1111/j.1461-0248.2005.00877.x.

[4] H. Held and T. Kleinen, "Detection of climate system bifurcations by degenerate fingerprinting," Geophys. Res. Lett., vol. 31, L23207, December 2004. doi: 10.1029/2004GL020972.

[5] V. Dakos, M. Scheffer, E.H. van Nes, V. Brovkin, V. Petoukhov, and H. Held, "Slowing down as an early warning signal for abrupt climate change," Proc. Natl. Acad. Sci. U.S.A., vol. 105, pp. 14308-14312, September 2008. doi: 10.1073/pnas.0802430105.

[6] E.A. Gopalakrishnan, Y. Sharma, T. John, P.S. Dutta, and R.I. Sujith, "Early warning signals for critical transitions in a thermoacoustic system," Sci. Rep., vol. 6, 35310, October 2016. doi: 10.1038/srep35310.

[7] A. Neumaier and T. Schneider, "Multivariate autoregressive and Ornstein-Uhlenbeck processes: Estimates for order, parameters, spectral information, and confidence regions," ACM Trans. Math. Software, 1998.

[8] A. Neumaier and T. Schneider, "Estimation of parameters and eigenmodes of multivariate autoregressive models," ACM Trans. Math. Software, vol. 27, pp. 27-57, March 2001. doi: $10.1145 / 382043.382304$.

[9] S.L. Pimm, "The complexity and stability of ecosystems," Nature, vol. 307, pp. 321-326, January 1984. doi: 10.1038/307321a0.

[10] E.H. van Nes and M. Scheffer, "Slow recovery from perturbations as a generic indicator of a nearby catastrophic shift," Am. Nat., vol. 169, pp. 738-747, June 2007. doi: 10.1086/516845.

[11] A.J. Veraart, E.J. Faassen, V. Dakos, E.H. van Nes, M. Lürling, and M. Scheffer, "Recovery rates reflect distance to a tipping point in a living system," Nature, vol. 481, pp. 357-359, January 2012. doi: 10.1038/nature10723.

[12] V. Dakos, E.H. van Nes, R. Donangelo, H. Fort, and M. Scheffer, "Spatial correlation as leading indicator of catastrophic shifts," Theor. Ecol., vol. 3, pp. 163-174, August 2010. doi: 10.1007/s12080-009-0060-6.

[13] J.M. Drake and B.D. Griffen, "Early warning signals of extinction in deteriorating environments," Nature, vol. 467, pp. 456-459, September 2010. doi: 10.1038/nature09389. 
[14] D.J. Fenn, M.A. Porter, S. Williams, M. McDonald, N.F. Johnson, and N.S. Jones, "Temporal evolution of financial-market correlations," Phys. Rev. E, vol. 84, 026109, August 2011. doi: 10.1103/PhysRevE.84.026109.

[15] L. Chen, R. Liu, Z.-P. Liu, M. Li, and K. Aihara, "Detecting early-warning signals for sudden deterioration of complex diseases by dynamical network biomarkers," Sci. Rep., vol. 2, 342, March 2012. doi: 10.1038/srep00342.

[16] I.A. van de Leemput, M. Wichers, A.O.J. Cramer, D. Borsboom, F. Tuerlinckx, P. Kuppens, E.H. van Nes, W. Viechtbauer, E.J. Giltay, S.H. Aggen, C. Derom, N. Jacobs, K.S. Kendler, H.L.J. van der Maas, M.C. Neale, F. Peeters, E. Thiery, P. Zachar, and M. Scheffer, "Critical slowing down as early warning for the onset and termination of depression," Proc. Natl. Acad. Sci. U.S.A., vol. 111, pp. 87-92, January 2014. doi: 10.1073/pnas.1312114110.

[17] W. Brock and S. Carpenter, "Variance as a leading indicator of regime shift in ecosystem services," Ecol. Soc., vol. 11, no. 2, 9, 2006. doi: 10.5751/ES-01777-110209.

[18] V. Dakos and J. Bascompte, "Critical slowing down as early warning for the onset of collapse in mutualistic communities," Proc. Natl. Acad. Sci. U.S.A., vol. 111, pp. 17546-17551, December 2014. doi: 10.1073 /pnas.1406326111.

[19] H. Moon and T.-C. Lu, "Network catastrophe: Self-organized patterns reveal both the instability and the structure of complex networks," Sci. Rep., vol. 5, 9450, March 2015. doi: $10.1038 /$ srep09450.

[20] O. Ledoit and M. Wolf, "A well-conditioned estimator for large-dimensional covariance matrices," J. Multivariate Anal., vol. 88, no. 2, pp. 365-411, 2004. doi: 10.1016/S0047259X(03)00096-4.

[21] A.R. Ives, B. Dennis, K.L. Cottingham, and S.R. Carpenter, "Estimating community stability and ecological interactions from time-series data," Ecol. Monogr., vol. 73, pp. 301-330, May 2003. doi: 10.1890/0012-9615(2003)073[0301:ECSAEI]2.0.CO;2.

[22] R. Biggs, S.R. Carpenter, and W.A. Brock, "Turning back from the brink: Detecting an impending regime shift in time to avert it," Proc. Natl. Acad. Sci. U.S.A., vol. 106, pp. 826-831, January 2009. doi: 10.1073/pnas.0811729106.

[23] T. Nakagawa, "Early warning signals by dynamical network markers," Bachelor's thesis, The University of Tokyo, February 2016. (in Japanese).

[24] S. Oya, K. Aihara, and Y. Hirata, "Forecasting abrupt changes in foreign exchange markets: Method using dynamical network marker," New J. Phys., vol. 16, 115015, November 2014. doi: 10.1088/1367-2630/16/11/115015.

[25] T. Nakagawa, M. Oku, and K. Aihara, "Early warning signals by dynamical network markers," SEISAN KENKYU, vol. 68, pp. 271-274, May 2016. (in Japanese). doi: 10.11188/ seisankenkyu.68.271.

[26] W.W.S. Wei, Time Series Analysis: Univariate and Multivariate Methods, Pearson Addison Wesley, 2nd ed., 2006.

[27] Z. Gajic and M.T.J. Qureshi, Lyapunov Matrix Equation in System Stability and Control, Dover Publications, 2008. (Republication of the Academic Press, San Diego, 1995 edition).

[28] B. Moore, "Principal component analysis in linear systems: Controllability, observability, and model reduction," IEEE Trans. Autom. Control, vol. 26, no. 1, pp. 17-32, 1981. doi: 10.1109/TAC.1981.1102568.

[29] C.W. Gardiner, Handbook of Stochastic Methods for Physics, Chemistry and the Natural Sciences, Springer-Verlag Berlin Heidelberg, 2nd ed., 1985.

[30] T. Sauer, "Numerical solution of stochastic differential equations in finance," in Handbook of Computational Finance (J.-C. Duan, W.K. Härdle, and J.E. Gentle, eds.), pp. 529-550, Springer Berlin Heidelberg, 2012. doi: 10.1007/978-3-642-17254-0_19.

[31] S.H. Strogatz, Nonlinear Dynamics and Chaos: With Applications to Physics, Biology, Chemistry, and Engineering, Perseus Books Publishing, 1994.

[32] V. Guttal and C. Jayaprakash, "Changing skewness: An early warning signal of regime shifts in ecosystems," Ecol. Lett., vol. 11, pp. 450-460, May 2008. doi: 10.1111/j.1461-0248.2008.01160.x. 
[33] V. Guttal and C. Jayaprakash, "Spatial variance and spatial skewness: Leading indicators of regime shifts in spatial ecological systems," Theor. Ecol., vol. 2, pp. 3-12, March 2009. doi: 10.1007/s12080-008-0033-1.

[34] V. Dakos, S.R. Carpenter, W.A. Brock, A.M. Ellison, V. Guttal, A.R. Ives, S. Kéfi, V. Livina, D.A. Seekell, E.H. van Nes, and M. Scheffer, "Methods for detecting early warnings of critical transitions in time series illustrated using simulated ecological data," PLOS ONE, vol. 7, e41010, July 2012. doi: 10.1371/journal.pone.0041010.

[35] J. Dall and M. Christensen, "Random geometric graphs," Phys. Rev. E, vol. 66, 016121, July 2002. doi: 10.1103/PhysRevE.66.016121.

[36] C.M. Bishop, Pattern Recognition and Machine Learning, Springer-Verlag New York, 2006.

[37] R. Liu, P. Chen, K. Aihara, and L. Chen, "Identifying early-warning signals of critical transitions with strong noise by dynamical network markers," Sci. Rep., vol. 5, 17501, December 2015. doi: $10.1038 / \operatorname{srep} 17501$.

[38] R. Graham and H. Haken, "Generalized thermodynamic potential for Markoff systems in detailed balance and far from thermal equilibrium," Z. Physik, vol. 243, no. 3, pp. 289-302, 1971. doi: 10.1007/BF01394858.

[39] M. Brics, J. Kaupuzs, and R. Mahnke, "How to solve Fokker-Planck equation treating mixed eigenvalue spectrum?," Condensed Matter Physics, vol. 16, no. 1, 13002, 2013. doi: 10.5488/CMP.16.13002.

[40] K. Kashima, "Noise response data reveal novel controllability Gramian for nonlinear network dynamics," Sci. Rep., vol. 6, 27300, June 2016. doi: 10.1038/srep27300.

[41] P.J. Menck, J. Heitzig, N. Marwan, and J. Kurths, "How basin stability complements the linearstability paradigm," Nature Phys., vol. 9, pp. 89-92, January 2013. doi: 10.1038/nphys2516.

[42] F. Hellmann, P. Schultz, C. Grabow, J. Heitzig, and J. Kurths, "Survivability of deterministic dynamical systems," Sci. Rep., vol. 6, 29654, July 2016. doi: 10.1038/srep29654.

[43] R. Wang, J.A. Dearing, P.G. Langdon, E. Zhang, X. Yang, V. Dakos, and M. Scheffer, "Flickering gives early warning signals of a critical transition to a eutrophic lake state," Nature, vol. 492, pp. 419-422, December 2012. doi: 10.1038/nature11655.

[44] X. Liu, R. Liu, X.-M. Zhao, and L. Chen, "Detecting early-warning signals of type 1 diabetes and its leading biomolecular networks by dynamical network biomarkers," BMC Med. Genomics, vol. 6, Suppl. 2, S8, May 2013. doi: 10.1186/1755-8794-6-S2-S8.

[45] M. Li, T. Zeng, R. Liu, and L. Chen, "Detecting tissue-specific early warning signals for complex diseases based on dynamical network biomarkers: Study of type 2 diabetes by cross-tissue analysis," Brief Bioinform., vol. 15, pp. 229-243, March 2014. doi: 10.1093/bib/bbt027.

[46] A.E. Teschendorff, X. Liu, H. Caren, S.M. Pollard, S. Beck, M. Widschwendter, and L. Chen, "The dynamics of DNA methylation covariation patterns in carcinogenesis," PLOS Comput. Biol., vol. 10, e1003709, July 2014. doi: 10.1371/journal.pcbi.1003709.

[47] P. Chen, R. Liu, L. Chen, and K. Aihara, "Identifying critical differentiation state of MCF-7 cells for breast cancer by dynamical network biomarkers," Front. Genet., vol. 6, 252, July 2015. doi: 10.3389 /fgene.2015.00252.

[48] A. Richard, L. Boullu, U. Herbach, A. Bonnafoux, V. Morin, E. Vallin, A. Guillemin, N. Papili Gao, R. Gunawan, J. Cosette, O. Arnaud, J.-J. Kupiec, T. Espinasse, S. Gonin-Giraud, and O. Gandrillon, "Single-cell-based analysis highlights a surge in cell-to-cell molecular variability preceding irreversible commitment in a differentiation process," PLOS Biol., vol. 14, e1002585, December 2016. doi: 10.1371/journal.pbio.1002585.

[49] R. Oldenburger, "Infinite powers of matrices and characteristic roots," Duke Math. J., vol. 6, no. 2, pp. 357-361, 1940. doi: 10.1215/S0012-7094-40-00627-5. 